\title{
Motor Symptoms as a Prodrome to Schizophrenia
}

\author{
Adam Schindzielorz, MD' ${ }^{1}$ Hunter Edwards, DO², Kelly Melvin, MD
}

ABSTRACT

Schizophrenia occurs in $1 \%$ of the population and severely impairs those afflicted.

Thereby, it is of the utmost importance to recognize and treat the disorder as early as possible. For the last several decades, research has attempted to elucidate various predictive factors to aid in identifying patients at high risk of developing a schizophrenia spectrum disorder. These factors include premorbid or prodromal movement symptoms. The literature has identified motor aberrations beginning early in life, in some cases even in infancy, ranging from neonatal hypotonicity to facial and upper body dyskinesia. These motor symptoms have been demonstrated to aid in predicting conversion to schizophrenia and carry prognostic importance for other outcomes. We present the case of a 34-year-old male who developed abnormal motor symptoms several years prior to the onset of psychosis. Our case builds upon the current body of literature by demonstrating the importance of spontaneous dyskinesia in evaluating populations at high risk for developing a schizophrenia spectrum illness, such as individuals with a family history of psychotic illness, schizotypal personality disorder, or other prodromal signs.

\section{KEYWORDS}

Early-onset, Prodrome, Schizophrenia, Psychotic Illness, Movement Disorder, Motor Symptoms, Dyskinesia

\section{INTRODUCTION}

Schizophrenia is a devastating and costly disease, costing sixty billion dollars per year in the United States. With nearly $1 \%$ of the world's population afflicted, new techniques for identifying at-risk individuals are critically important. ${ }^{1}$ Unfortunately, clinical detection often occurs after the disease has caused marked impairment. Evidence indicates that through early identification of core prodromal symptoms, rapid and aggressive intervention can significantly improve prognosis. ${ }^{2}$

Outside of psychopharmacologic exposure, motoric symptoms have been identified as a key component of the illness. For example, Eugene Bleuler observed irregular movements in schizophrenic patients before the advent of modern psychopharmacologic agents. ${ }^{3}$ Similarly, contemporary studies have reported spontaneous dyskinesias in medication naïve patients. ${ }^{4}$ Motor symptoms do not appear to be limited to the active phase schizophrenia either, as literature supports the existence of motoric abnormalities before the onset of psychosis, even as early as childhood. ${ }^{5}$ Additionally, motor symptoms have validity in predicting transition to psychotic illness in high-risk groups (first-degree relative with history of psychotic illness, schizotypal personality disorder, or prodromal signs) and are correlated with disease severity and functional outcomes. ${ }^{6,7}$

\section{CASE REPORT}

The patient was a 34-year-old male initially treated for attention deficit hyperactivity disorder, an unspecified depressive illness, and severe social anxiety. Symptoms were initially managed with 
alprazolam, amphetamine-dextroamphetamine, and doxepin, which were stopped when he moved in with his parents and established with a new physician after living alone during his early twenties. Soon after, he developed facial tics including perioral twitching, rapid eye blinking, intermittent anteroand retro torticollis, rotation of the upper torso, and asymmetric shoulder shrugging. He also developed severe right-sided facial pain.

He was seen by neurology between 2013 and 2014, during which head computed tomography, magnetic resonance imaging, and angiography were performed and found to be without abnormality. Carbamazepine, gabapentin, and cyclobenzaprine were started for diagnoses of blepharospasm and an unspecified tic disorder. Later, he followed up with a psychiatrist who added sertraline and clonazepam to his regimen for depressive and anxious symptoms.

In 2014, the patient transferred care to local neurology and psychiatry services. Quetiapine $25 \mathrm{mg}$ was added by neurology to treat continued motor symptoms and stopped shortly thereafter with concern that symptoms were psychogenic. After 2016, the patient began to follow up exclusively with psychiatry. At that time, diagnoses included major depressive disorder, attention deficit hyperactivity disorder, social anxiety, and panic disorders. He was treated with duloxetine $90 \mathrm{mg}$ daily and clonazepam, later changed to lorazepam $1 \mathrm{mg}$ three times daily. He was also re-trialed on amphetaminedextroamphetamine for impaired concentration, which was stopped after the patient developed auditory hallucinations; these resolved shortly thereafter.

In 2017, the patient's family notified the psychiatry clinic that he had been suffering from a recurrence of perceptual disturbances, now with associated paranoid ideation. Recommendations were given for emergent evaluation in the emergency department and psychiatric hospitalization. The patient ceased coming to appointments until later that year when his family called again, reporting similar symptoms. The patient was also now experiencing command hallucinations instructing him to harm himself, leading to the patient fleeing his home.
Several weeks later, the patient arrived for a scheduled outpatient appointment. He reported ongoing psychotic symptoms, including both commenting and command auditory hallucinations involving multiple voices, as well as ongoing delusional ideation involving the FBI planting recording devices in his home. Speech and thought were disorganized, and previously reported motor symptoms were appreciated on examination, noted by the patient to have never resolved. The patient was started on olanzapine, titrated to $15 \mathrm{mg}$ daily, and lorazepam and duloxetine continued. His diagnosis was modified to schizophrenia, paranoid type. On subsequent visits, the patient demonstrated significant improvement in both perceptual and cognitive disturbances and his motoric symptoms.

\section{DISCUSSION}

Schizophrenia commonly devastates a patient's ability to function. With an average age of onset in the third decade of life, schizophrenia strikes when an individual would otherwise be establishing independence. ${ }^{8}$ Because diagnosis is clinical, symptoms are often already causing marked impairment by the time they are recognized. With the impact of schizophrenia being so costly for patients and the healthcare system, research has focused on establishing reliable markers to identify at-risk populations to allow for earlier intervention.

Prodromal movement symptoms hold diagnostic and prognostic value and are associated with increased risk of transitioning to psychosis and worsened prognosis. ${ }^{9,10}$ Before the advent of antipsychotic medications, movement disorders were recognized as commonplace in schizophrenia and innate to the disease. ${ }^{3}$ Now, they are mostly viewed as iatrogenic. However, recent research has identified motor symptoms as widely present in high-risk patients and a critical component in their evaluation. ${ }^{11,12}$ For example, Mittal et al. examined the relationship between movement disorders in adolescents with schizotypal personality disorder, a diagnosis associated with schizophrenia. The authors found that patients at risk for psychosis possessed greater degrees of motor abnormality in the period mds.marshall.edu/mjm

(c) 2022 Marshall Journal of Medicine
Marshall Journal of Medicine

Volume 8 Issue 1 
prior to the onset of psychotic symptoms. ${ }^{6}$ Moreover, Peralta et al. examined treatment-naïve patients with schizophrenia and found a prevalence of motor abnormalities in $66 \%$, demonstrating the existence of motor symptoms outside of antipsychotic use. ${ }^{12}$

Though we cannot rule out that our patient's development of motor symptoms was not secondary to a comorbid neurologic illness, their onset in the context of cognitive deterioration, psychosis, and negative neurologic work-up makes these symptoms suspect for being part of prodromal schizophrenia. We posit that our patient's case is likely representative of many others in that because his initial presenting symptoms were non-specific, they could have been attributed to other causes. Misdiagnosis and subsequent mistreatment are lost opportunities for early intervention, making the recognition of prodromal symptomatology critical to obtaining early access to care.

\section{CONCLUSION}

Ultimately, we feel that our patient represents a case where both neurocognitive and neuro-motor symptomatology manifested prior to the onset of his eventual psychosis. This case fits the view of schizophrenia being neurodevelopmental in nature. Given the dearth of prospective studies, this patient's case serves to add to the volume of knowledge identifying possible biomarkers for screening and diagnosing premorbid schizophrenia.

\section{COMPETING INTERESTS}

The authors declare that they have no competing interests.

\section{CONSENT}

Verbal consent was granted by the patient to write and publish this article.

\section{AUTHOR AFFILIATIONS}

1. Marshall University Joan C. Edwards School of Medicine, Huntington, West Virginia

2. Mountain Area Health Education Center, Asheville, NC

\section{REFERENCES}

1. Leiberman JA, Dixon LB, Goldman HH. Early detection and intervention in schizophrenia a new therapeutic model. JAMA. 2013 Aug 31;310(7):689-90.

2. Haas GL, Garratt LS, Sweeney JA. Delay to first antipsychotic medication in schizophrenia: Impact on symptomatology and clinical course of illness. J Psychiatr Res. 1998 MayAug;32(3-4):151-9.

3. Walther S, Werner S. Motor symptoms and schizophrenia. Neuropsychobiology. 2012;66(2):77-92.

4. Gervin M, Brown S, Lane A, Clarke M, Waddington J, Larkin C, O'Callaghan E. Spontaneous abnormal involuntary movements in firstepisode schizophrenia and schizophreniform disorder: baseline rate in a group of patients from an irish catchment area. Am J Psychiatry. 1998 Sep;155(9):1202-6.

5. Schiffman J, Walker E, Ekstrom M, Schulsinger F, Sorensen H, Mednick S. Childhood videotapes social and neuromotor precursors of schizophrenia. Am J Psychiatry. 2004 Nov;161(11):2021-7.

6. Mittal VA, Neumann C, Saczawa M, Walker EF. Longitudinal progression of movement abnormalities in relation to psychotic symptoms in adolescents at high risk of schizophrenia. Arch Gen Psychiatry. 2008;65(2):165-171.

7. Carrion R, McLaughlin D, Goldberg T, Auther A, Olsen $R$, Olvet $D$, et al. Prediction of functional outcome in individuals at clinical high risk for psychosis. JAMA Psychiatry. 2013;70(11):11331142.

8. Meltzer $\mathrm{H}$, Rabinowitz J, Lee $\mathrm{M}$, Cola P, Ranjan $\mathrm{R}$, Findling R, Thompson P. Age at onset and gender of schizophrenic patients in relation to neuroleptic resistance. Am J Psychiatry. 1997 Apr;154(4):475-482.

9. Nelson B, Yuen HP, Wood SJ, Lin A, 
Spiliotacopoulos D, Bruzner A, et al. Longterm follow-up of a group at ultra-high risk ("prodromal") for psychosis. JAMA Psychiatry. 2013;70(8):793-802.

10. Hirjak D, Meyer-Lindenberg A, Kubera K, Thomann P, Wolf R. Motor dysfunction as research domain in the period preceding manifest schizophrenia: a systematic review. Neurosci Biobehav Reviews. 2018 Apr;87:87-105.

11. Klosterkötter J, Hellmich M, Steinmeyer EM, Schultze-Lutter F. Diagnosing Schizophrenia in the initial prodromal phase. Arch Gen Psychiatry. 2001 Feb;58(2):158-64.

12. Peralta V, Campos MS, De Jalon EG, Cuesta MJ. Motor behavior abnormalities in drug-naive patients with schizophrenia spectrum disorders. Mov Disord. 2010 Jun 15;25(8):1068-76. 